\section{International Scientific Journal Theoretical \& Applied Science}

\author{
p-ISSN: 2308-4944 (print) e-ISSN: 2409-0085 (online) \\ Year: $2018 \quad$ Issue: $01 \quad$ Volume: 57 \\ Published: $30.01 .2018 \quad$ http://T-Science.org
}

Denis Chemezov

M.Sc.Eng., Corresponding Member of International Academy of Theoretical and Applied Sciences, Lecturer of Vladimir Industrial College, Russian Federation chemezov-da@yandex.ru

Tatyana Lukyanova Lecturer of Vladimir Industrial College, Russian Federation

SECTION 2. Applied mathematics. Mathematical modeling.

\title{
AERODYNAMIC AND HYDRODYNAMIC FLOW PARAMETERS OF GASES AND LIQUIDS
}

Abstract: Changes of the parameters values of transient flow of gases and liquids when increasing pressure are presented in the article. Comparison of densities, flow velocities, vorticities, dynamic pressures, the Prandtl numbers, turbulent energies and other motion parameters of liquids and gases was performed.

Key words: liquid, gas, flow.

Language: English

Citation: Chemezov D, Lukyanova T (2018) AERODYNAMIC AND HYDRODYNAMIC FLOW PARAMETERS OF GASES AND LIQUIDS. ISJ Theoretical \& Applied Science, 01 (57): 101-107.

Soi: http://s-o-i.org/1.1/TAS-01-57-18 Doi: crossef https://dx.doi.org/10.15863/TAS.2018.01.57.18

\section{Introduction}

In the work [1] it is given representation about character of water flow in a straight pipeline. Transient flow is characterized by vortices formation of different intensity in fluid [2]. Flow of liquids and gases in a some volume in large part has the same character. However, a change of density during flow (filling of the volume) of gases leads to a significant increasing of pressure, temperature, flow velocity, vorticity and etc. Density of liquid is almost constant and values of hydrodynamic parameters will depend on value of fluid movement per unit time and a cross sectional area of the pipeline. In this article by a numerical simulation there were defined aerodynamic and hydrodynamic parameters of transient flow of liquids and gases. These calculated values of parameters will allow to choose material, overall dimensions and other characteristics of a device for transporting of fluids.

\section{Materials and methods}

Aerodynamic and hydrodynamic parameters of liquids and gases and their dependencies when increasing of pressure are determined. For research there were taken the following fluids:

1. Acetone (liquid and gas);

2. Air (gas);

3. Ammonia (liquid and gas);

4. Argon (liquid and gas);

5. Butane (gas);

6. Carbon dioxide (gas);

7. Chlorine (gas);
8. Ethane (liquid and gas);

9. Ethanol (liquid and gas);

10. Ethylene (liquid and gas);

11. Fluorine (gas);

12. Helium (gas);

13. Hydrogen (gas);

14. Krypton (gas);

15. Methane (liquid and gas);

16. Methanol (liquid and gas);

17. Neon (gas);

18. Nitrogen (liquid and gas);

19. Oxygen (liquid and gas);

20. Propane (liquid and gas);

21. Propylene (gas);

22. R22 (liquid) [3];

23. R123 (liquid) [4];

24. R134a (liquid) [5];

25. RC318 (liquid) [6];

26. Water (liquid);

27. Xenon (gas).

The process of flow of liquids and gases was carried out by the same conditions. Flow direction was constant for liquids and gases. Due to lower density, mass flow rate of gas was reduced in 10 times in comparison with mass flow rate of liquids.

\section{Results and discussion}

Comparing of flow parameters of liquids and gases is presented in the summary table 1 . Min and max are minimum and maximum values of aerodynamic and hydrodynamic parameters of liquids and gases, respectively. 


\section{Impact Factor:}

\begin{tabular}{|c|c|c|}
\hline ISRA (India) & 344 & SIS (USA) \\
\hline ISI (Dub & & РИНЦ (Russia) \\
\hline GIF & & ESJI (KZ) \\
\hline & $=1$. & SJIF $($ Morocco $)=\mathbf{2}$ \\
\hline
\end{tabular}

ICV (Poland)

$=6.630$

PIF (India)

$=1.940$

IBI (India)
From considered liquids the highest density has RC318, from gases the highest density has xenon. Density and temperature of liquid are almost not changed when increasing of hydraulic pressure in the conditions of flow. For gases there are characteristically significant increasing of velocity flow, density and temperature when increasing of pressure.

Table 1

Flow parameters of gases and liquids.

\begin{tabular}{|c|c|c|c|c|}
\hline \multicolumn{5}{|c|}{ Acetone (liquid) } \\
\hline Pressure, $\mathrm{Pa}$ & Temperature, $\mathrm{K}$ & Density, $\mathrm{kg} / \mathrm{m}^{3}$ & Velocity, $\mathrm{m} / \mathrm{s}$ & Radial velocity, $\mathrm{m} / \mathrm{s}$ \\
\hline $101325 / 273077.81$ & $293.2 / 293.22$ & $792.57 / 792.6$ & $0 / 0.378$ & $-0.023 / 0.055$ \\
\hline Dynamic pressure, $\mathrm{Pa}$ & Shear stress, $\mathrm{Pa}$ & Specific heat, $\mathrm{J} /(\mathrm{kg} \cdot \mathrm{K})$ & Dynamic viscosity, $\mathrm{Pa} \cdot \mathrm{s}$ & Vorticity, $1 / \mathrm{s}$ \\
\hline $0 / 56.66$ & $0 / 4.68 \times 10^{-4}$ & $2160.2 / 2160.3$ & $0.0003 / 0.0003$ & $0.005 / 28.714$ \\
\hline Prandtl number & Mach number & Fluid thermal conductivity, $\mathrm{W} /(\mathrm{m} \cdot \mathrm{K})$ & Turbulent viscosity, Pa·s & Turbulent time, $\mathrm{s}$ \\
\hline $4.2409535 / 4.241596$ & - & $0.1615 / 0.1615$ & $2.8616 \times 10^{-5} / 539.1951$ & $0.029 / 59.089$ \\
\hline Turbulent length, $\mathrm{m}$ & Turbulent energy, J/kg & Turbulent dissipation, W/kg & Operative temperature, $\mathrm{K}$ & PMV [7] \\
\hline $8 \times 10^{-4} / 0.266$ & $6.789 \times 10^{-9} / 21.809$ & $1.15 \times 10^{-10} / 62.92$ & $293.22 / 293.22$ & $-1.41 /-0.81$ \\
\hline \multicolumn{5}{|c|}{ Acetone (gas) } \\
\hline Pressure, $\mathrm{Pa}$ & Temperature, $\mathrm{K}$ & Density, $\mathrm{kg} / \mathrm{m}^{3}$ & Velocity, $\mathrm{m} / \mathrm{s}$ & Radial velocity, $\mathrm{m} / \mathrm{s}$ \\
\hline $101325 / 364382.24$ & $287 / 293.21$ & $2.49 / 8.77$ & $0 / 12.446$ & $-0.424 / 1.487$ \\
\hline Dynamic pressure, $\mathrm{Pa}$ & Shear stress, Pa & Specific heat, $\mathrm{J} /(\mathrm{kg} \cdot \mathrm{K})$ & Dynamic viscosity, Pa·s & Vorticity, $1 / \mathrm{s}$ \\
\hline $0 / 194.01$ & $0 / 2.03 \times 10^{-4}$ & $1296.9 / 1886.9$ & $7.237 \times 10^{-6} / 7.3954 \times 10^{-6}$ & $0.082 / 953.962$ \\
\hline Prandtl number & Mach number & Fluid thermal conductivity, $\mathrm{W} /(\mathrm{m} \cdot \mathrm{K})$ & Turbulent viscosity, $\mathrm{Pa} \cdot \mathrm{s}$ & Turbulent time, $\mathrm{s}$ \\
\hline $0.8590031 / 1.2587339$ & $0 / 0.06$ & $0.0108 / 0.0111$ & $2.8616 \times 10^{-6} / 39.4804$ & $0.002 / 6.443$ \\
\hline Turbulent length, $m$ & Turbulent energy, J/kg & Turbulent dissipation, W/kg & Operative temperature, $\mathrm{K}$ & PMV \\
\hline $8 \times 10^{-4} / 0.212$ & $5.709 \times 10^{-7} / 4025.732$ & $8.86 \times 10^{-8} / 210586.71$ & $287.26 / 291.69$ & $-5.59 /-0.91$ \\
\hline \multicolumn{5}{|c|}{ Air (gas) } \\
\hline Pressure, $\mathrm{Pa}$ & Temperature, $\mathrm{K}$ & Density, $\mathrm{kg} / \mathrm{m}^{3}$ & Velocity, $\mathrm{m} / \mathrm{s}$ & Radial velocity, $\mathrm{m} / \mathrm{s}$ \\
\hline $101325 / 456625.1$ & $279.99 / 293.2$ & $1.29 / 5.57$ & $0 / 22.644$ & $-0.467 / 2.823$ \\
\hline Dynamic pressure, $\mathrm{Pa}$ & Shear stress, $\mathrm{Pa}$ & Specific heat, $\mathrm{J} /(\mathrm{kg} \cdot \mathrm{K})$ & Dynamic viscosity, $\mathrm{Pa} \cdot \mathrm{s}$ & Vorticity, $1 / \mathrm{s}$ \\
\hline $0 / 333.54$ & $0 / 4.98 \times 10^{-4}$ & $1013.1 / 2093.3$ & $1.7378 \times 10^{-5} / 1.8102 \times 10^{-5}$ & $0.07 / 1755.568$ \\
\hline Prandtl number & Mach number & Fluid thermal conductivity, $\mathrm{W} /(\mathrm{m} \cdot \mathrm{K})$ & Turbulent viscosity, $\mathrm{Pa} \cdot \mathrm{s}$ & Turbulent time, $\mathrm{s}$ \\
\hline $0.710799 / 1.4687759$ & $0 / 0.07$ & $0.0247 / 0.0254$ & $2.8616 \times 10^{-6} / 25.7753$ & $0.002 / 4.057$ \\
\hline Turbulent length, $\mathrm{m}$ & Turbulent energy, J/kg & Turbulent dissipation, $\mathrm{W} / \mathrm{kg}$ & Operative temperature, $\mathrm{K}$ & PMV \\
\hline $8 \times 10^{-4} / 0.171$ & $1.44 \times 10^{-6} / 8564.652$ & $3.55 \times 10^{-7} / 875062.31$ & $280.46 / 290.85$ & $-9.06 /-1.18$ \\
\hline \multicolumn{5}{|c|}{ Ammonia (liquid) } \\
\hline Pressure, $\mathrm{Pa}$ & Temperature, $\mathrm{K}$ & Density, $\mathrm{kg} / \mathrm{m}^{3}$ & Velocity, $\mathrm{m} / \mathrm{s}$ & Radial velocity, $\mathrm{m} / \mathrm{s}$ \\
\hline $101325 / 337636.99$ & 293.2/293.21 & $609.44 / 609.45$ & $0 / 0.492$ & $-0.028 / 0.07$ \\
\hline Dynamic pressure, $\mathrm{Pa}$ & Shear stress, $\mathrm{Pa}$ & Specific heat, $\mathrm{J} /(\mathrm{kg} \cdot \mathrm{K})$ & Dynamic viscosity, $\mathrm{Pa} \cdot \mathrm{s}$ & Vorticity, $1 / \mathrm{s}$ \\
\hline $0 / 73.63$ & $0 / 3.99 \times 10^{-4}$ & $4745.6 / 4745.6$ & $0.0001 / 0.0001$ & $0.004 / 37.392$ \\
\hline Prandtl number & Mach number & Fluid thermal conductivity, $\mathrm{W} /(\mathrm{m} \cdot \mathrm{K})$ & Turbulent viscosity, $\mathrm{Pa} \cdot \mathrm{s}$ & Turbulent time, $\mathrm{s}$ \\
\hline $1.3134004 / 1.3134267$ & - & $0.4997 / 0.4997$ & $2.8616 \times 10^{-5} / 535.5151$ & $0.022 / 45.436$ \\
\hline Turbulent length, $\mathrm{m}$ & Turbulent energy, J/kg & Turbulent dissipation, $\mathrm{W} / \mathrm{kg}$ & Operative temperature, $\mathrm{K}$ & PMV \\
\hline $8 \times 10^{-4} / 0.266$ & $1.148 \times 10^{-8} / 36.382$ & $2.53 \times 10^{-10} / 135.59$ & $293.2 / 293.21$ & $-1.55 /-0.82$ \\
\hline \multicolumn{5}{|c|}{ Ammonia (gas) } \\
\hline Pressure, $\mathrm{Pa}$ & Temperature, $\mathrm{K}$ & Density, $\mathrm{kg} / \mathrm{m}^{3}$ & Velocity, $\mathrm{m} / \mathrm{s}$ & Radial velocity, $\mathrm{m} / \mathrm{s}$ \\
\hline $101325 / 555840.42$ & $279.99 / 293.2$ & $0.77 / 4.01$ & $0 / 37.824$ & $-0.769 / 4.714$ \\
\hline Dynamic pressure, $\mathrm{Pa}$ & Shear stress, $\mathrm{Pa}$ & Specific heat, $\mathrm{J} /(\mathrm{kg} \cdot \mathrm{K})$ & Dynamic viscosity, Pa·s & Vorticity, $1 / \mathrm{s}$ \\
\hline $0 / 556.23$ & $0 / 5.13 \times 10^{-4}$ & $2172.5 / 4024.4$ & $9.613 \times 10^{-6} / 1.0066 \times 10^{-5}$ & $0.006 / 2963.638$ \\
\hline Prandtl number & Mach number & Fluid thermal conductivity, $\mathrm{W} /(\mathrm{m} \cdot \mathrm{K})$ & Turbulent viscosity, $\mathrm{Pa} \cdot \mathrm{s}$ & Turbulent time, $\mathrm{s}$ \\
\hline $0.9275258 / 1.7181831$ & $0 / 0.09$ & $0.0221 / 0.0231$ & $2.8616 \times 10^{-6} / 23.9603$ & $0.001 / 2.921$ \\
\hline Turbulent length, $\mathrm{m}$ & Turbulent energy, J/kg & Turbulent dissipation, $\mathrm{W} / \mathrm{kg}$ & Operative temperature, $\mathrm{K}$ & PMV \\
\hline $8 \times 10^{-4} / 0.164$ & $2.778 \times 10^{-6} / 17514.341$ & $9.51 \times 10^{-7} / 2783322.37$ & $280.37 / 291.29$ & $-9.38 /-1.15$ \\
\hline \multicolumn{5}{|c|}{ Argon (liquid) } \\
\hline Pressure, $\mathrm{Pa}$ & Temperature, $\mathrm{K}$ & Density, $\mathrm{kg} / \mathrm{m}^{3}$ & Velocity, $\mathrm{m} / \mathrm{s}$ & Radial velocity, $\mathrm{m} / \mathrm{s}$ \\
\hline $101325 / 230689.1$ & $293.12 / 293.2$ & $1164.07 / 1164.07$ & $0 / 0.257$ & $-0.014 / 0.037$ \\
\hline Dynamic pressure, $\mathrm{Pa}$ & Shear stress, $\mathrm{Pa}$ & Specific heat, $\mathrm{J} /(\mathrm{kg} \cdot \mathrm{K})$ & Dynamic viscosity, $\mathrm{Pa} \cdot \mathrm{s}$ & Vorticity, $1 / \mathrm{s}$ \\
\hline $0 / 38.54$ & $0 / 1.85 \times 10^{-4}$ & $1332.4 / 1332.4$ & $0.0001 / 0.0001$ & $5.728 \times 10^{-4} / 19.437$ \\
\hline Prandtl number & Mach number & Fluid thermal conductivity, $\mathrm{W} /(\mathrm{m} \cdot \mathrm{K})$ & Turbulent viscosity, $\mathrm{Pa} \cdot \mathrm{s}$ & Turbulent time, $\mathrm{s}$ \\
\hline $1.7436429 / 1.7436429$ & - & $0.0832 / 0.0832$ & $2.8616 \times 10^{-5} / 532.9398$ & $0.043 / 86.783$ \\
\hline Turbulent length, $\mathrm{m}$ & Turbulent energy, J/kg & Turbulent dissipation, $\mathrm{W} / \mathrm{kg}$ & Operative temperature, $\mathrm{K}$ & PMV \\
\hline $8 \times 10^{-4} / 0.266$ & $3.147 \times 10^{-9} / 9.879$ & $3.63 \times 10^{-11} / 19.19$ & $293.14 / 293.16$ & $-1.24 /-0.82$ \\
\hline \multicolumn{5}{|c|}{ Argon (gas) } \\
\hline Pressure, $\mathrm{Pa}$ & Temperature, $\mathrm{K}$ & Density, $\mathrm{kg} / \mathrm{m}^{3}$ & Velocity, $\mathrm{m} / \mathrm{s}$ & Radial velocity, $\mathrm{m} / \mathrm{s}$ \\
\hline $101325 / 424904.75$ & $279.99 / 293.2$ & $1.75 / 7.13$ & $0 / 16.857$ & $-0.429 / 2.092$ \\
\hline Dynamic pressure, $\mathrm{Pa}$ & Shear stress, $\mathrm{Pa}$ & Specific heat, $\mathrm{J} /(\mathrm{kg} \cdot \mathrm{K})$ & Dynamic viscosity, $\mathrm{Pa} \cdot \mathrm{s}$ & Vorticity, $1 / \mathrm{s}$ \\
\hline $0 / 253.37$ & $0 / 4.32 \times 10^{-4}$ & $529 / 1321.7$ & $2.1517 \times 10^{-5} / 2.2401 \times 10^{-5}$ & $0.033 / 1314.836$ \\
\hline Prandtl number & Mach number & Fluid thermal conductivity, $\mathrm{W} /(\mathrm{m} \cdot \mathrm{K})$ & Turbulent viscosity, $\mathrm{Pa} \cdot \mathrm{s}$ & Turbulent time, $\mathrm{s}$ \\
\hline
\end{tabular}




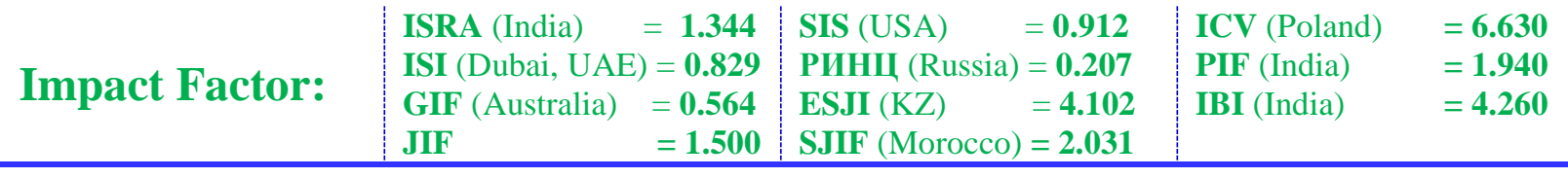

\begin{tabular}{|c|c|c|c|c|}
\hline $0.6741774 / 1.6843888$ & $0 / 0.06$ & $0.0169 / 0.0173$ & $2.8616 \times 10^{-6} / 31.947$ & $0.002 / 5.192$ \\
\hline Turbulent length, $\mathrm{m}$ & Turbulent energy, J/kg & Turbulent dissipation, W/kg & Operative temperature, $\mathrm{K}$ & PMV \\
\hline $8 \times 10^{-4} / 0.188$ & $8.794 \times 10^{-7} / 5780.122$ & $1.69 \times 10^{-7} / 423356.34$ & $280.54 / 290.76$ & $-8.83 /-1.16$ \\
\hline \multicolumn{5}{|c|}{ Butane (gas) } \\
\hline Pressure, $\mathrm{Pa}$ & Temperature, $\mathrm{K}$ & Density, $\mathrm{kg} / \mathrm{m}^{3}$ & Velocity, $\mathrm{m} / \mathrm{s}$ & Radial velocity, $\mathrm{m} / \mathrm{s}$ \\
\hline $101325 / 366278.5$ & $288.24 / 293.21$ & $2.48 / 8.8$ & $0 / 12.51$ & $-0.434 / 1.499$ \\
\hline Dynamic pressure, $\mathrm{Pa}$ & Shear stress, $\mathrm{Pa}$ & Specific heat, $\mathrm{J} /(\mathrm{kg} \cdot \mathrm{K})$ & Dynamic viscosity, $\mathrm{Pa} \cdot \mathrm{s}$ & Vorticity, $1 / \mathrm{s}$ \\
\hline $0 / 195.26$ & $0 / 2.02 \times 10^{-4}$ & $1638.1 / 2205.3$ & $7.25 \times 10^{-6} / 7.3836 \times 10^{-6}$ & $0.079 / 957.771$ \\
\hline Prandtl number & Mach number & Fluid thermal conductivity, $\mathrm{W} /(\mathrm{m} \cdot \mathrm{K})$ & Turbulent viscosity, $\mathrm{Pa} \cdot \mathrm{s}$ & Turbulent time, $\mathrm{s}$ \\
\hline $0.7985167 / 1.0780602$ & $0 / 0.06$ & $0.0148 / 0.0151$ & $2.8616 \times 10^{-6} / 40.0093$ & $0.002 / 6.479$ \\
\hline Turbulent length, $\mathrm{m}$ & Turbulent energy, J/kg & Turbulent dissipation, W/kg & Operative temperature, $\mathrm{K}$ & PMV \\
\hline $8 \times 10^{-4} / 0.213$ & $5.647 \times 10^{-7} / 4070.98$ & $8.72 \times 10^{-8} / 212874.76$ & $288.45 / 292.01$ & $-5.11 /-0.84$ \\
\hline \multicolumn{5}{|c|}{ Carbon dioxide (gas) } \\
\hline Pressure, $\mathrm{Pa}$ & Temperature, $\mathrm{K}$ & Density, $\mathrm{kg} / \mathrm{m}^{3}$ & Velocity, $\mathrm{m} / \mathrm{s}$ & Radial velocity, $\mathrm{m} / \mathrm{s}$ \\
\hline $101325 / 410978.05$ & $280.1 / 293.2$ & $1.91 / 7.61$ & $0 / 15.667$ & $-0.471 / 1.92$ \\
\hline Dynamic pressure, $\mathrm{Pa}$ & Shear stress, $\mathrm{Pa}$ & Specific heat, $\mathrm{J} /(\mathrm{kg} \cdot \mathrm{K})$ & Dynamic viscosity, $\mathrm{Pa} \cdot \mathrm{s}$ & Vorticity, $1 / \mathrm{s}$ \\
\hline $0 / 239.73$ & $0 / 3.26 \times 10^{-4}$ & $858.9 / 1601.2$ & $1.3984 \times 10^{-5} / 1.4647 \times 10^{-5}$ & $0.052 / 1222.446$ \\
\hline Prandtl number & Mach number & Fluid thermal conductivity, $\mathrm{W} /(\mathrm{m} \cdot \mathrm{K})$ & Turbulent viscosity, $\mathrm{Pa} \cdot \mathrm{s}$ & Turbulent time, $\mathrm{s}$ \\
\hline $0.7032722 / 1.314071$ & $0 / 0.06$ & $0.0171 / 0.0175$ & $2.8616 \times 10^{-6} / 35.2204$ & $0.002 / 5.524$ \\
\hline Turbulent length, $\mathrm{m}$ & Turbulent energy, J/kg & Turbulent dissipation, W/kg & Operative temperature, $\mathrm{K}$ & PMV \\
\hline $8 \times 10^{-4} / 0.198$ & $7.769 \times 10^{-7} / 5306.59$ & $1.41 \times 10^{-7} / 349105.89$ & $281.19 / 290.38$ & $-8.22 /-1.23$ \\
\hline \multicolumn{5}{|c|}{ Chlorine (gas) } \\
\hline Pressure, $\mathrm{Pa}$ & Temperature, $\mathrm{K}$ & Density, $\mathrm{kg} / \mathrm{m}^{3}$ & Velocity, $\mathrm{m} / \mathrm{s}$ & Radial velocity, $\mathrm{m} / \mathrm{s}$ \\
\hline $101325 / 330813.8$ & $281.31 / 293.22$ & $3.02 / 9.82$ & $0 / 10.231$ & $\begin{array}{l}-0.447 / 1.209 \\
-0\end{array}$ \\
\hline Dynamic pressure, $\mathrm{Pa}$ & Shear stress, $\mathrm{Pa}$ & Specific heat, $\mathrm{J} /(\mathrm{kg} \cdot \mathrm{K})$ & Dynamic viscosity, Pa·s & Vorticity, $1 / \mathrm{s}$ \\
\hline $0 / 162.4$ & $0 / 2.41 \times 10^{-4}$ & $483.1 / 976$ & $1.2506 \times 10^{-5} / 1.3064 \times 10^{-5}$ & $0.07 / 782.105$ \\
\hline Prandtl number & Mach number & Fluid thermal conductivity, $\mathrm{W} /(\mathrm{m} \cdot \mathrm{K})$ & Turbulent viscosity, $\mathrm{Pa} \cdot \mathrm{s}$ & Turbulent time, $\mathrm{s}$ \\
\hline $0.6972721 / 1.409706$ & $0 / 0.05$ & $0.0087 / 0.0088$ & $2.8616 \times 10^{-6} / 41.7238$ & $0.003 / 7.122$ \\
\hline Turbulent length, $\mathrm{m}$ & Turbulent energy, J/kg & Turbulent dissipation, $\mathrm{W} / \mathrm{kg}$ & Operative temperature, $\mathrm{K}$ & PMV \\
\hline $8 \times 10^{-4} / 0.22$ & $4.673 \times 10^{-7} / 3127.628$ & $6.56 \times 10^{-8} / 136309.3$ & $282.27 / 290.28$ & $-7.48 /-1.18$ \\
\hline \multicolumn{5}{|c|}{ Ethane (liquid) } \\
\hline Pressure, $\mathrm{Pa}$ & Temperature, $\mathrm{K}$ & Density, $\mathrm{kg} / \mathrm{m}^{3}$ & Velocity, $\mathrm{m} / \mathrm{s}$ & Radial velocity, $\mathrm{m} / \mathrm{s}$ \\
\hline $101325 / 406539.98$ & $293.18 / 293.2$ & $457.85 / 457.85$ & $0 / 0.655$ & $\begin{array}{l}-0.039 / 0.094 \\
\end{array}$ \\
\hline Dynamic pressure, $\mathrm{Pa}$ & Shear stress, Pa & Specific heat, $\mathrm{J} /(\mathrm{kg} \cdot \mathrm{K})$ & Dynamic viscosity, $\mathrm{Pa} \cdot \mathrm{s}$ & Vorticity, $1 / \mathrm{s}$ \\
\hline $0 / 98.07$ & $0 / 4.1 \times 10^{-4}$ & $2921.6 / 2921.6$ & $8.1812 \times 10^{-5} / 8.1812 \times 10^{-5}$ & $0.006 / 49.632$ \\
\hline Prandtl number & Mach number & Fluid thermal conductivity, $\mathrm{W} /(\mathrm{m} \cdot \mathrm{K})$ & Turbulent viscosity, $\mathrm{Pa} \cdot \mathrm{s}$ & Turbulent time, $\mathrm{s}$ \\
\hline $2.1261514 / 2.1261514$ & - & $0.1124 / 0.1124$ & $2.8616 \times 10^{-5} / 537.1098$ & $0.017 / 34.134$ \\
\hline Turbulent length, $\mathrm{m}$ & Turbulent energy, J/kg & Turbulent dissipation, $\mathrm{W} / \mathrm{kg}$ & Operative temperature, $\mathrm{K}$ & PMV \\
\hline $8 \times 10^{-4} / 0.266$ & $2.034 \times 10^{-8} / 64.858$ & $5.96 \times 10^{-10} / 322.76$ & $293.18 / 293.2$ & $-1.70 /-0.82$ \\
\hline \multicolumn{5}{|c|}{ Ethane (gas) } \\
\hline Pressure, $\mathrm{Pa}$ & Temperature, $\mathrm{K}$ & Density, $\mathrm{kg} / \mathrm{m}^{3}$ & Velocity, $\mathrm{m} / \mathrm{s}$ & Radial velocity, $\mathrm{m} / \mathrm{s}$ \\
\hline $101325 / 457975.39$ & $281.01 / 293.2$ & $1.33 / 5.79$ & $0 / 21.941$ & $-0.504 / 2.754$ \\
\hline Dynamic pressure, $\mathrm{Pa}$ & Shear stress, $\mathrm{Pa}$ & Specific heat, $\mathrm{J} /(\mathrm{kg} \cdot \mathrm{K})$ & Dynamic viscosity, Pa.s & Vorticity, $1 / \mathrm{s}$ \\
\hline $0 / 322.33$ & $0 / 3.38 \times 10^{-4}$ & $1750.6 / 2874.4$ & $8.7775 \times 10^{-6} / 9.125 \times 10^{-6}$ & $0.069 / 1691.727$ \\
\hline Prandtl number & Mach number & Fluid thermal conductivity, $\mathrm{W} /(\mathrm{m} \cdot \mathrm{K})$ & Turbulent viscosity, $\mathrm{Pa} \cdot \mathrm{s}$ & Turbulent time, $\mathrm{s}$ \\
\hline $0.7765416 / 1.3136242$ & $0 / 0.07$ & $0.0193 / 0.02$ & $2.8616 \times 10^{-6} / 26.3385$ & $0.002 / 4.225$ \\
\hline Turbulent length, $\mathrm{m}$ & Turbulent energy, J/kg & Turbulent dissipation, W/kg & Operative temperature, $\mathrm{K}$ & PMV \\
\hline $8 \times 10^{-4} / 0.172$ & $1.328 \times 10^{-6} / 8196.684$ & $3.14 \times 10^{-7} / 813500.84$ & $281.99 / 290.95$ & $-8.01 /-1.15$ \\
\hline \multicolumn{5}{|c|}{ Ethanol (liquid) } \\
\hline Pressure, $\mathrm{Pa}$ & Temperature, $\mathrm{K}$ & Density, $\mathrm{kg} / \mathrm{m}^{3}$ & Velocity, $\mathrm{m} / \mathrm{s}$ & Radial velocity, $\mathrm{m} / \mathrm{s}$ \\
\hline $101325 / 273119.12$ & $293.2 / 293.23$ & $790.51 / 790.53$ & $0 / 0.379$ & $-0.023 / 0.055$ \\
\hline Dynamic pressure, $\mathrm{Pa}$ & Shear stress, $\mathrm{Pa}$ & Specific heat, $\mathrm{J} /(\mathrm{kg} \cdot \mathrm{K})$ & Dynamic viscosity, $\mathrm{Pa} \cdot \mathrm{s}$ & Vorticity, $1 / \mathrm{s}$ \\
\hline $0 / 56.8$ & $0 / 8.87 \times 10^{-4}$ & $2398 / 2398.3$ & $0.0011 / 0.0011$ & $0.005 / 28.694$ \\
\hline Prandtl number & Mach number & Fluid thermal conductivity, $\mathrm{W} /(\mathrm{m} \cdot \mathrm{K})$ & Turbulent viscosity, $\mathrm{Pa} \cdot \mathrm{s}$ & Turbulent time, $\mathrm{s}$ \\
\hline 18.119794/18.126087 & - & $0.1512 / 0.1512$ & $2.8616 \times 10^{-5} / 539.0227$ & $0.029 / 58.935$ \\
\hline Turbulent length, $\mathrm{m}$ & Turbulent energy, J/kg & Turbulent dissipation, $\mathrm{W} / \mathrm{kg}$ & Operative temperature, $\mathrm{K}$ & PMV \\
\hline $8 \times 10^{-4} / 0.266$ & $6.824 \times 10^{-9} / 21.909$ & $1.16 \times 10^{-10} / 63.36$ & $293.22 / 293.23$ & $-1.41 /-0.81$ \\
\hline \multicolumn{5}{|c|}{ Ethanol (gas) } \\
\hline Pressure, $\mathrm{Pa}$ & Temperature, $\mathrm{K}$ & Density, $\mathrm{kg} / \mathrm{m}^{3}$ & Velocity, $\mathrm{m} / \mathrm{s}$ & Radial velocity, $\mathrm{m} / \mathrm{s}$ \\
\hline $101325 / 405988.39$ & $285.24 / 293.2$ & $2 / 7.8$ & $0 / 15.249$ & $-0.478 / 1.862$ \\
\hline Dynamic pressure, $\mathrm{Pa}$ & Shear stress, $\mathrm{Pa}$ & Specific heat, $\mathrm{J} /(\mathrm{kg} \cdot \mathrm{K})$ & Dynamic viscosity, $\mathrm{Pa} \cdot \mathrm{s}$ & Vorticity, $1 / \mathrm{s}$ \\
\hline $0 / 233.72$ & $0 / 2.42 \times 10^{-4}$ & $1428.8 / 2189.2$ & $8.2198 \times 10^{-6} / 8.4551 \times 10^{-6}$ & $0.067 / 1192.605$ \\
\hline Prandtl number & Mach number & Fluid thermal conductivity, $\mathrm{W} /(\mathrm{m} \cdot \mathrm{K})$ & Turbulent viscosity, $\mathrm{Pa} \cdot \mathrm{s}$ & Turbulent time, $\mathrm{s}$ \\
\hline $0.8075278 / 1.2704423$ & $0 / 0.06$ & $0.0142 / 0.0146$ & $2.8616 \times 10^{-6} / 36.6393$ & $0.002 / 5.713$ \\
\hline Turbulent length, $\mathrm{m}$ & Turbulent energy, $\mathrm{J} / \mathrm{kg}$ & Turbulent dissipation, W/kg & Operative temperature, $\mathrm{K}$ & PMV \\
\hline $8 \times 10^{-4} / 0.202$ & $7.263 \times 10^{-7} / 5181.581$ & $1.27 \times 10^{-7} / 329219.05$ & $285.55 / 291.31$ & $-6.41 /-1.01$ \\
\hline \multicolumn{5}{|c|}{ Ethylene (liquid) } \\
\hline Pressure, $\mathrm{Pa}$ & Temperature, $\mathrm{K}$ & Density, $\mathrm{kg} / \mathrm{m}^{3}$ & Velocity, $\mathrm{m} / \mathrm{s}$ & Radial velocity, $\mathrm{m} / \mathrm{s}$ \\
\hline $101325 / 389794.47$ & $293.16 / 293.2$ & $481.14 / 481.14$ & $0 / 0.623$ & $-0.037 / 0.09$ \\
\hline Dynamic pressure, $\mathrm{Pa}$ & Shear stress, $\mathrm{Pa}$ & Specific heat, $\mathrm{J} /(\mathrm{kg} \cdot \mathrm{K})$ & Dynamic viscosity, Pa $\cdot \mathrm{s}$ & Vorticity, $1 / \mathrm{s}$ \\
\hline
\end{tabular}




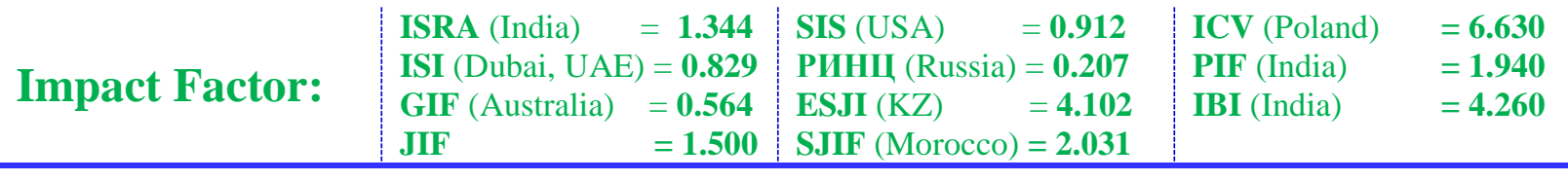

\begin{tabular}{|c|c|c|c|c|}
\hline $0 / 93.31$ & $0 / 4.12 \times 10^{-4}$ & $2750.3 / 2750.3$ & $9.1291 \times 10^{-5} / 9.1291 \times 10^{-5}$ & $0.007 / 47.194$ \\
\hline Prandtl number & Mach number & Fluid thermal conductivity, $\mathrm{W} /(\mathrm{m} \cdot \mathrm{K})$ & Turbulent viscosity, $\mathrm{Pa} \cdot \mathrm{s}$ & Turbulent time, $\mathrm{s}$ \\
\hline $1.9205816 / 1.9205816$ & - & $0.1307 / 0.1307$ & $2.8616 \times 10^{-5} / 537.8207$ & $0.017 / 35.869$ \\
\hline Turbulent length, $\mathrm{m}$ & Turbulent energy, J/kg & Turbulent dissipation, W/kg & Operative temperature, $\mathrm{K}$ & PMV \\
\hline $8 \times 10^{-4} / 0.266$ & $1.842 \times 10^{-8} / 58.891$ & $5.14 \times 10^{-10} / 279.26$ & $293.17 / 293.19$ & $-1.68 /-0.82$ \\
\hline \multicolumn{5}{|c|}{ Ethylene (gas) } \\
\hline Pressure, $\mathrm{Pa}$ & Temperature, $\mathrm{K}$ & Density, $\mathrm{kg} / \mathrm{m}^{3}$ & Velocity, $\mathrm{m} / \mathrm{s}$ & Radial velocity, $\mathrm{m} / \mathrm{s}$ \\
\hline $101325 / 463688.31$ & $279.99 / 293.2$ & $1.25 / 5.49$ & $0 / 23.333$ & $-0.501 / 2.904$ \\
\hline Dynamic pressure, $\mathrm{Pa}$ & Shear stress, $\mathrm{Pa}$ & Specific heat, $\mathrm{J} /(\mathrm{kg} \cdot \mathrm{K})$ & Dynamic viscosity, $\mathrm{Pa} \cdot \mathrm{s}$ & Vorticity, $1 / \mathrm{s}$ \\
\hline $0 / 342.98$ & $0 / 3.78 \times 10^{-4}$ & $1518.6 / 2647.1$ & $9.7537 \times 10^{-6} / 1.0179 \times 10^{-5}$ & $0.086 / 1800.252$ \\
\hline Prandtl number & Mach number & Fluid thermal conductivity, $\mathrm{W} /(\mathrm{m} \cdot \mathrm{K})$ & Turbulent viscosity, $\mathrm{Pa} \cdot \mathrm{s}$ & Turbulent time, $\mathrm{s}$ \\
\hline $0.7791445 / 1.3581254$ & $0 / 0.07$ & $0.0184 / 0.0193$ & $2.8616 \times 10^{-6} / 25.6195$ & $0.002 / 3.993$ \\
\hline Turbulent length, $\mathrm{m}$ & Turbulent energy, $\mathrm{J} / \mathrm{kg}$ & Turbulent dissipation, $\mathrm{W} / \mathrm{kg}$ & Operative temperature, $\mathrm{K}$ & PMV \\
\hline $8 \times 10^{-4} / 0.17$ & $1.487 \times 10^{-6} / 8933.909$ & $3.72 \times 10^{-7} / 939667.93$ & $280.53 / 290.75$ & $-8.77 /-1.21$ \\
\hline \multicolumn{5}{|c|}{ Fluorine (gas) } \\
\hline Pressure, $\mathrm{Pa}$ & Temperature, $\mathrm{K}$ & Density, $\mathrm{kg} / \mathrm{m}^{3}$ & Velocity, $\mathrm{m} / \mathrm{s}$ & Radial velocity, $\mathrm{m} / \mathrm{s}$ \\
\hline $101325 / 433086.27$ & $279.99 / 293.2$ & $1.67 / 6.93$ & $0 / 17.575$ & $-0.421 / 2.185$ \\
\hline Dynamic pressure, $\mathrm{Pa}$ & Shear stress, $\mathrm{Pa}$ & Specific heat, $\mathrm{J} /(\mathrm{kg} \cdot \mathrm{K})$ & Dynamic viscosity, Pa·s & Vorticity, $1 / \mathrm{s}$ \\
\hline $0 / 262.34$ & $0 / 4.53 \times 10^{-4}$ & $832.6 / 1671.5$ & $2.2349 \times 10^{-5} / 2.3408 \times 10^{-5}$ & $0.017 / 1359.94$ \\
\hline Prandtl number & Mach number & Fluid thermal conductivity, $\mathrm{W} /(\mathrm{m} \cdot \mathrm{K})$ & Turbulent viscosity, $\mathrm{Pa} \cdot \mathrm{s}$ & Turbulent time, $\mathrm{s}$ \\
\hline $0.7116161 / 1.4285958$ & $0 / 0.06$ & $0.0263 / 0.0269$ & $2.8616 \times 10^{-6} / 30.9442$ & $0.002 / 5.038$ \\
\hline Turbulent length, $\mathrm{m}$ & Turbulent energy, J/kg & Turbulent dissipation, W/kg & Operative temperature, $\mathrm{K}$ & PMV \\
\hline $8 \times 10^{-4} / 0.185$ & $9.339 \times 10^{-7} / 6104.408$ & $1.85 \times 10^{-7} / 470904.42$ & $280.48 / 290.45$ & $-8.89 /-1.24$ \\
\hline \multicolumn{5}{|c|}{ Helium (gas) } \\
\hline Pressure, $\mathrm{Pa}$ & Temperature, $\mathrm{K}$ & Density, $\mathrm{kg} / \mathrm{m}^{3}$ & Velocity, $\mathrm{m} / \mathrm{s}$ & Radial velocity, $\mathrm{m} / \mathrm{s}$ \\
\hline $101325 / 803929.48$ & $279.99 / 293.2$ & $0.22 / 1.4$ & $0 / 130.398$ & $-6.932 / 13.659$ \\
\hline Dynamic pressure, $\mathrm{Pa}$ & Shear stress, $\mathrm{Pa}$ & Specific heat, $\mathrm{J} /(\mathrm{kg} \cdot \mathrm{K})$ & Dynamic viscosity, $\mathrm{Pa} \cdot \mathrm{s}$ & Vorticity, $1 / \mathrm{s}$ \\
\hline $0 / 1871.67$ & $0 / 2.12 \times 10^{-3}$ & $5028.9 / 12566.6$ & $1.926 \times 10^{-5} / 1.995 \times 10^{-5}$ & $0.22 / 9090.682$ \\
\hline Prandtl number & Mach number & Fluid thermal conductivity, $\mathrm{W} /(\mathrm{m} \cdot \mathrm{K})$ & Turbulent viscosity, $\mathrm{Pa} \cdot \mathrm{s}$ & Turbulent time, $\mathrm{s}$ \\
\hline $0.6572041 / 1.6421951$ & $0 / 0.15$ & $0.1477 / 0.1524$ & $2.8616 \times 10^{-6} / 12.4479$ & $8.69 \times 10^{-4} / 1.034$ \\
\hline Turbulent length, $\mathrm{m}$ & Turbulent energy, J/kg & Turbulent dissipation, W/kg & Operative temperature, $\mathrm{K}$ & PMV \\
\hline $8 \times 10^{-4} / 0.13$ & $2.218 \times 10^{-5} / 102745.56$ & $2.15 \times 10^{-5} / 5.94 \times 10^{7}$ & $280.28 / 292.58$ & $-9.93 /-1.11$ \\
\hline \multicolumn{5}{|c|}{ Hydrogen (gas) } \\
\hline Pressure, $\mathrm{Pa}$ & Temperature, $\mathrm{K}$ & Density, $\mathrm{kg} / \mathrm{m}^{3}$ & Velocity, $\mathrm{m} / \mathrm{s}$ & Radial velocity, $\mathrm{m} / \mathrm{s}$ \\
\hline $101325 / 1091988.06$ & $279.99 / 293.2$ & $0.12 / 1.01$ & $0 / 224.332$ & $-9.791 / 30.617$ \\
\hline Dynamic pressure, $\mathrm{Pa}$ & Shear stress, $\mathrm{Pa}$ & Specific heat, $\mathrm{J} /(\mathrm{kg} \cdot \mathrm{K})$ & Dynamic viscosity, $\mathrm{Pa} \cdot \mathrm{s}$ & Vorticity, $1 / \mathrm{s}$ \\
\hline $0 / 3117.81$ & $0 / 2.17 \times 10^{-3}$ & 13111.4/27511.1 & $8.6119 \times 10^{-6} / 8.9414 \times 10^{-6}$ & $1.076 / 15949.458$ \\
\hline Prandtl number & Mach number & Fluid thermal conductivity, $\mathrm{W} /(\mathrm{m} \cdot \mathrm{K})$ & Turbulent viscosity, $\mathrm{Pa} \cdot \mathrm{s}$ & Turbulent time, $\mathrm{s}$ \\
\hline $0.6453035 / 1.3540073$ & $0 / 0.2$ & $0.1757 / 0.1824$ & $2.8616 \times 10^{-6} / 14.78$ & $8.097 \times 10^{-4} / 0.742$ \\
\hline Turbulent length, $\mathrm{m}$ & Turbulent energy, $\mathrm{J} / \mathrm{kg}$ & Turbulent dissipation, W/kg & Operative temperature, $\mathrm{K}$ & PMV \\
\hline $8 \times 10^{-4} / 0.138$ & $4.305 \times 10^{-5} / 225569.14$ & $5.8 \times 10^{-5} / 1.98 \times 10^{8}$ & $280.22 / 292.78$ & $-10.11 /-1.1$ \\
\hline \multicolumn{5}{|c|}{ Krypton (gas) } \\
\hline Pressure, $\mathrm{Pa}$ & Temperature, $\mathrm{K}$ & Density, $\mathrm{kg} / \mathrm{m}^{3}$ & Velocity, $\mathrm{m} / \mathrm{s}$ & Radial velocity, $\mathrm{m} / \mathrm{s}$ \\
\hline $101325 / 302070.72$ & $279.99 / 293.23$ & $3.55 / 10.62$ & $0 / 8.765$ & $-0.45 / 1.011$ \\
\hline Dynamic pressure, $\mathrm{Pa}$ & Shear stress, $\mathrm{Pa}$ & Specific heat, $\mathrm{J} /(\mathrm{kg} \cdot \mathrm{K})$ & Dynamic viscosity, Pa·s & Vorticity, 1/s \\
\hline $0 / 141.53$ & $0 / 3.09 \times 10^{-4}$ & $253.4 / 635.8$ & $2.3605 \times 10^{-5} / 2.4643 \times 10^{-5}$ & $0.058 / 656.323$ \\
\hline Prandtl number & Mach number & Fluid thermal conductivity, $\mathrm{W} /(\mathrm{m} \cdot \mathrm{K})$ & Turbulent viscosity, $\mathrm{Pa} \cdot \mathrm{s}$ & Turbulent time, $\mathrm{s}$ \\
\hline $0.6693651 / 1.6797399$ & $0 / 0.05$ & $0.0089 / 0.0091$ & $2.8616 \times 10^{-6} / 43.4197$ & $0.003 / 7.671$ \\
\hline Turbulent length, $\mathrm{m}$ & Turbulent energy, $\mathrm{J} / \mathrm{kg}$ & Turbulent dissipation, W/kg & Operative temperature, $\mathrm{K}$ & PMV \\
\hline $8 \times 10^{-4} / 0.228$ & $4.029 \times 10^{-7} / 2566.96$ & $5.25 \times 10^{-8} / 97108.21$ & $280.67 / 289.91$ & $-8.3 /-1.22$ \\
\hline \multicolumn{5}{|c|}{ Methane (liquid) } \\
\hline Pressure, $\mathrm{Pa}$ & Temperature, $\mathrm{K}$ & Density, $\mathrm{kg} / \mathrm{m}^{3}$ & Velocity, $\mathrm{m} / \mathrm{s}$ & Radial velocity, $\mathrm{m} / \mathrm{s}$ \\
\hline $101325 / 487770.13$ & $293.05 / 293.2$ & $356.4 / 356.4$ & $0 / 0.841$ & $-0.05 / 0.121$ \\
\hline Dynamic pressure, $\mathrm{Pa}$ & Shear stress, Pa & Specific heat, $\mathrm{J} /(\mathrm{kg} \cdot \mathrm{K})$ & Dynamic viscosity, $\mathrm{Pa} \cdot \mathrm{s}$ & Vorticity, $1 / \mathrm{s}$ \\
\hline $0 / 125.95$ & $0 / 4.3 \times 10^{-4}$ & $4077.4 / 4077.5$ & $5.4455 \times 10^{-5} / 5.4455 \times 10^{-5}$ & $0.01 / 63.659$ \\
\hline Prandtl number & Mach number & Fluid thermal conductivity, $\mathrm{W} /(\mathrm{m} \cdot \mathrm{K})$ & Turbulent viscosity, $\mathrm{Pa} \cdot \mathrm{s}$ & Turbulent time, $\mathrm{s}$ \\
\hline $1.734554 / 1.734554$ & - & $0.128 / 0.128$ & $2.8616 \times 10^{-5} / 538.3689$ & $0.013 / 26.57$ \\
\hline Turbulent length, $\mathrm{m}$ & Turbulent energy, J/kg & Turbulent dissipation, W/kg & Operative temperature, $\mathrm{K}$ & PMV \\
\hline $8 \times 10^{-4} / 0.266$ & $3.358 \times 10^{-8} / 107.539$ & $1.26 \times 10^{-9} / 689.07$ & $293.07 / 293.13$ & $-1.88 /-0.83$ \\
\hline \multicolumn{5}{|c|}{ Methane (gas) } \\
\hline Pressure, $\mathrm{Pa}$ & Temperature, $\mathrm{K}$ & Density, $\mathrm{kg} / \mathrm{m}^{3}$ & Velocity, $\mathrm{m} / \mathrm{s}$ & Radial velocity, $\mathrm{m} / \mathrm{s}$ \\
\hline $101325 / 451219.43$ & $279.99 / 293.2$ & $0.73 / 3.06$ & $0 / 39.362$ & $-1.485 / 4.941$ \\
\hline Dynamic pressure, $\mathrm{Pa}$ & Shear stress, $\mathrm{Pa}$ & Specific heat, $\mathrm{J} /(\mathrm{kg} \cdot \mathrm{K})$ & Dynamic viscosity, $\mathrm{Pa} \cdot \mathrm{s}$ & Vorticity, $1 / \mathrm{s}$ \\
\hline $0 / 570.57$ & $0 / 7.86 \times 10^{-4}$ & $2215.6 / 4188.6$ & $1.0486 \times 10^{-5} / 1.0915 \times 10^{-5}$ & $0.315 / 3032.419$ \\
\hline Prandtl number & Mach number & Fluid thermal conductivity, $\mathrm{W} /(\mathrm{m} \cdot \mathrm{K})$ & Turbulent viscosity, $\mathrm{Pa} \cdot \mathrm{s}$ & Turbulent time, $\mathrm{s}$ \\
\hline $0.702232 / 1.3275826$ & $0 / 0.09$ & $0.0329 / 0.0345$ & $5.0779 \times 10^{-9} / 17.118$ & $0.006 / 2.238$ \\
\hline Turbulent length, $\mathrm{m}$ & Turbulent energy, $\mathrm{J} / \mathrm{kg}$ & Turbulent dissipation, $\mathrm{W} / \mathrm{kg}$ & Operative temperature, $\mathrm{K}$ & PMV \\
\hline $2.902 \times 10^{-5} / 0.138$ & $4.734 \times 10^{-6} / 17653.214$ & $2.12 \times 10^{-6} / 2862731.13$ & $280.62 / 293.19$ & $-9.29 /-0.59$ \\
\hline \multicolumn{5}{|c|}{ Methanol (liquid) } \\
\hline Pressure, $\mathrm{Pa}$ & Temperature, $\mathrm{K}$ & Density, $\mathrm{kg} / \mathrm{m}^{3}$ & Velocity, $\mathrm{m} / \mathrm{s}$ & Radial velocity, $\mathrm{m} / \mathrm{s}$ \\
\hline
\end{tabular}




\begin{tabular}{|c|c|c|c|c|c|c|}
\hline Impact Factor: & $\begin{array}{l}\text { ISRA (India) } \\
\text { ISI (Dubai, UAF } \\
\text { GIF (Australia) } \\
\text { JIF }\end{array}$ & $\begin{array}{l}=1.344 \\
=0.829 \\
=0.564 \\
=1.500\end{array}$ & $\begin{array}{l}\text { SIS (USA) } \\
\text { PИНЦ (Russia } \\
\text { ESJI (KZ) } \\
\text { SJIF (Morocce }\end{array}$ & $\begin{array}{l}=0.912 \\
=0.207 \\
=\mathbf{4 . 1 0 2} \\
=\mathbf{2 . 0 3 1}\end{array}$ & $\begin{array}{l}\text { ICV (Poland) } \\
\text { PIF (India) } \\
\text { IBI (India) }\end{array}$ & $\begin{array}{l}=6.630 \\
=1.940 \\
=4.260\end{array}$ \\
\hline
\end{tabular}

\begin{tabular}{|c|c|c|c|c|}
\hline $101325 / 280950.19$ & $293.2 / 293.23$ & $791.51 / 791.54$ & $0 / 0.379$ & $-0.022 / 0.054$ \\
\hline Dynamic pressure, $\mathrm{Pa}$ & Shear stress, $\mathrm{Pa}$ & Specific heat, $\mathrm{J} /(\mathrm{kg} \cdot \mathrm{K})$ & Dynamic viscosity, $\mathrm{Pa} \cdot \mathrm{s}$ & Vorticity, $1 / \mathrm{s}$ \\
\hline $0 / 56.71$ & $0 / 6.16 \times 10^{-4}$ & $2505.2 / 2505.4$ & $0.0006 / 0.0006$ & $0.003 / 28.746$ \\
\hline Prandtl number & Mach number & Fluid thermal conductivity, $\mathrm{W} /(\mathrm{m} \cdot \mathrm{K})$ & Turbulent viscosity, $\mathrm{Pa} \cdot \mathrm{s}$ & Turbulent time, $\mathrm{s}$ \\
\hline $7.2250349 / 7.2268248$ & - & $0.1922 / 0.1922$ & $2.8616 \times 10^{-5} / 536.2664$ & $0.029 / 59.01$ \\
\hline Turbulent length, $\mathrm{m}$ & Turbulent energy, J/kg & Turbulent dissipation, W/kg & Operative temperature, $\mathrm{K}$ & PMV \\
\hline $8 \times 10^{-4} / 0.266$ & $6.807 \times 10^{-9} / 21.638$ & $1.15 \times 10^{-10} / 62.2$ & $293.22 / 293.23$ & $-1.41 /-0.81$ \\
\hline \multicolumn{5}{|c|}{ Methanol (gas) } \\
\hline Pressure, $\mathrm{Pa}$ & Temperature, $\mathrm{K}$ & Density, $\mathrm{kg} / \mathrm{m}^{3}$ & Velocity, $\mathrm{m} / \mathrm{s}$ & Radial velocity, $\mathrm{m} / \mathrm{s}$ \\
\hline $101325 / 445382.05$ & $280.77 / 293.2$ & $1.41 / 5.99$ & $0 / 20.978$ & $-0.506 / 2.615$ \\
\hline Dynamic pressure, $\mathrm{Pa}$ & Shear stress, Pa & Specific heat, $\mathrm{J} /(\mathrm{kg} \cdot \mathrm{K})$ & Dynamic viscosity, Pa.s & Vorticity, $1 / \mathrm{s}$ \\
\hline $0 / 313.32$ & $0 / 3.32 \times 10^{-4}$ & $1490 / 2503.9$ & $9.0676 \times 10^{-6} / 9.5031 \times 10^{-6}$ & $0.027 / 1633.678$ \\
\hline Prandtl number & Mach number & Fluid thermal conductivity, $\mathrm{W} /(\mathrm{m} \cdot \mathrm{K})$ & Turbulent viscosity, $\mathrm{Pa} \cdot \mathrm{s}$ & Turbulent time, $\mathrm{s}$ \\
\hline $0.9670902 / 1.6768456$ & $0 / 0.07$ & $0.0136 / 0.0142$ & $2.8616 \times 10^{-6} / 27.7621$ & $0.002 / 4.368$ \\
\hline Turbulent length, m & Turbulent energy, $\mathrm{J} / \mathrm{kg}$ & Turbulent dissipation, W/kg & Operative temperature, $\mathrm{K}$ & PMV \\
\hline $8 \times 10^{-4} / 0.176$ & $1.243 \times 10^{-6} / 7693.616$ & $2.84 \times 10^{-7} / 711279.11$ & $281.8 / 290.85$ & $-8.12 /-1.17$ \\
\hline \multicolumn{5}{|c|}{ Neon (gas) } \\
\hline Pressure, $\mathrm{Pa}$ & Temperature, $\mathrm{K}$ & Density, $\mathrm{kg} / \mathrm{m}^{3}$ & Velocity, $\mathrm{m} / \mathrm{s}$ & Radial velocity, $\mathrm{m} / \mathrm{s}$ \\
\hline $101325 / 514882.28$ & $279.99 / 293.2$ & $0.91 / 4.37$ & $0 / 32.475$ & $-0.759 / 4.031$ \\
\hline Dynamic pressure, $\mathrm{Pa}$ & Shear stress, Pa & Specific heat, $\mathrm{J} /(\mathrm{kg} \cdot \mathrm{K})$ & Dynamic viscosity, Pa.s & Vorticity, $1 / \mathrm{s}$ \\
\hline $0 / 482.51$ & $0 / 8.23 \times 10^{-4}$ & $1038.9 / 2599.1$ & $2.9538 \times 10^{-5} / 3.0607 \times 10^{-5}$ & $0.035 / 2542.451$ \\
\hline Prandtl number & Mach number & Fluid thermal conductivity, $\mathrm{W} /(\mathrm{m} \cdot \mathrm{K})$ & Turbulent viscosity, $\mathrm{Pa} \cdot \mathrm{s}$ & Turbulent time, $\mathrm{s}$ \\
\hline $0.6643545 / 1.6620547$ & $0 / 0.08$ & $0.0461 / 0.0474$ & $2.8616 \times 10^{-6} / 24.6251$ & $0.002 / 3.198$ \\
\hline Turbulent length, $\mathrm{m}$ & Turbulent energy, J/kg & Turbulent dissipation, W/kg & Operative temperature, $\mathrm{K}$ & PMV \\
\hline $8 \times 10^{-4} / 0.167$ & $2.317 \times 10^{-6} / 14131.592$ & $7.25 \times 10^{-7} / 1945504.68$ & $280.45 / 291.56$ & $-9.27 /-1.05$ \\
\hline \multicolumn{5}{|c|}{ Nitrogen (liquid) } \\
\hline Pressure, $\mathrm{Pa}$ & Temperature, $\mathrm{K}$ & Density, $\mathrm{kg} / \mathrm{m}^{3}$ & Velocity, $\mathrm{m} / \mathrm{s}$ & Radial velocity, $\mathrm{m} / \mathrm{s}$ \\
\hline $101325 / 309968.11$ & $293.05 / 293.2$ & $684.47 / 684.47$ & $0 / 0.438$ & $-0.025 / 0.063$ \\
\hline Dynamic pressure, $\mathrm{Pa}$ & Shear stress, $\mathrm{Pa}$ & Specific heat, $\mathrm{J} /(\mathrm{kg} \cdot \mathrm{K})$ & Dynamic viscosity, $\mathrm{Pa} \cdot \mathrm{s}$ & Vorticity, $1 / \mathrm{s}$ \\
\hline $0 / 65.59$ & $0 / 2.59 \times 10^{-4}$ & $2344.6 / 2344.7$ & $7.3155 \times 10^{-5} / 7.3155 \times 10^{-5}$ & $0.003 / 33.36$ \\
\hline Prandtl number & Mach number & Fluid thermal conductivity, $\mathrm{W} /(\mathrm{m} \cdot \mathrm{K})$ & Turbulent viscosity, $\mathrm{Pa} \cdot \mathrm{s}$ & Turbulent time, $\mathrm{s}$ \\
\hline $1.7394612 / 1.7394612$ & - & $0.0986 / 0.0986$ & $2.8616 \times 10^{-5} / 536.0209$ & $0.025 / 51.028$ \\
\hline Turbulent length, $\mathrm{m}$ & Turbulent energy, J/kg & Turbulent dissipation, W/kg & Operative temperature, $\mathrm{K}$ & PMV \\
\hline $8 \times 10^{-4} / 0.266$ & $9.103 \times 10^{-9} / 28.906$ & $1.78 \times 10^{-10} / 96.04$ & $293.07 / 293.12$ & $-1.53 /-0.83$ \\
\hline \multicolumn{5}{|c|}{ Nitrogen (gas) } \\
\hline Pressure, $\mathrm{Pa}$ & Temperature, $\mathrm{K}$ & Density, $\mathrm{kg} / \mathrm{m}^{3}$ & Velocity, $\mathrm{m} / \mathrm{s}$ & Radial velocity, $\mathrm{m} / \mathrm{s}$ \\
\hline $101325 / 458495.98$ & $279.99 / 293.2$ & $1.25 / 5.41$ & $0 / 23.455$ & $-0.494 / 2.899$ \\
\hline Dynamic pressure, $\mathrm{Pa}$ & Shear stress, $\mathrm{Pa}$ & Specific heat, $\mathrm{J} /(\mathrm{kg} \cdot \mathrm{K})$ & Dynamic viscosity, $\mathrm{Pa} \cdot \mathrm{s}$ & Vorticity, 1/s \\
\hline $0 / 346.38$ & $0 / 5.05 \times 10^{-4}$ & $1047.4 / 2184.8$ & $1.6824 \times 10^{-5} / 1.7454 \times 10^{-5}$ & $0.079 / 1806.965$ \\
\hline Prandtl number & Mach number & Fluid thermal conductivity, $\mathrm{W} /(\mathrm{m} \cdot \mathrm{K})$ & Turbulent viscosity, $\mathrm{Pa} \cdot \mathrm{s}$ & Turbulent time, $\mathrm{s}$ \\
\hline $0.720611 / 1.5032227$ & $0 / 0.07$ & $0.0243 / 0.025$ & $2.8616 \times 10^{-6} / 25.4253$ & $0.002 / 3.942$ \\
\hline Turbulent length, $\mathrm{m}$ & Turbulent energy, J/kg & Turbulent dissipation, W/kg & Operative temperature, $\mathrm{K}$ & PMV \\
\hline $8 \times 10^{-4} / 0.17$ & $1.526 \times 10^{-6} / 8973.901$ & $3.87 \times 10^{-7} / 944569.8$ & $280.45 / 290.9$ & $-9.08 /-1.17$ \\
\hline \multicolumn{5}{|c|}{ Oxygen (liquid) } \\
\hline Pressure, $\mathrm{Pa}$ & Temperature, $\mathrm{K}$ & Density, $\mathrm{kg} / \mathrm{m}^{3}$ & Velocity, $\mathrm{m} / \mathrm{s}$ & Radial velocity, $\mathrm{m} / \mathrm{s}$ \\
\hline $101325 / 253489.12$ & $293.14 / 293.2$ & $951.33 / 951.33$ & $0 / 0.315$ & $-0.018 / 0.045$ \\
\hline Dynamic pressure, $\mathrm{Pa}$ & Shear stress, $\mathrm{Pa}$ & Specific heat, $\mathrm{J} /(\mathrm{kg} \cdot \mathrm{K})$ & Dynamic viscosity, $\mathrm{Pa} \cdot \mathrm{s}$ & Vorticity, $1 / \mathrm{s}$ \\
\hline $0 / 47.16$ & $0 / 2.11 \times 10^{-4}$ & $1999.4 / 1999.4$ & $9.4276 \times 10^{-5} / 9.4276 \times 10^{-5}$ & $0.002 / 23.835$ \\
\hline Prandtl number & Mach number & Fluid thermal conductivity, $\mathrm{W} /(\mathrm{m} \cdot \mathrm{K})$ & Turbulent viscosity, $\mathrm{Pa} \cdot \mathrm{s}$ & Turbulent time, $\mathrm{s}$ \\
\hline $1.8605807 / 1.8605807$ & - & $0.1013 / 0.1013$ & $2.8616 \times 10^{-5} / 535.2102$ & $0.035 / 70.923$ \\
\hline Turbulent length, $\mathrm{m}$ & Turbulent energy, J/kg & Turbulent dissipation, $\mathrm{W} / \mathrm{kg}$ & Operative temperature, $\mathrm{K}$ & PMV \\
\hline $8 \times 10^{-4} / 0.266$ & $4.712 \times 10^{-9} / 14.914$ & $6.64 \times 10^{-11} / 35.59$ & $293.15 / 293.17$ & $-1.34 /-0.82$ \\
\hline \multicolumn{5}{|c|}{ Oxygen (gas) } \\
\hline Pressure, $\mathrm{Pa}$ & Temperature, $\mathrm{K}$ & Density, $\mathrm{kg} / \mathrm{m}^{3}$ & Velocity, $\mathrm{m} / \mathrm{s}$ & Radial velocity, $\mathrm{m} / \mathrm{s}$ \\
\hline $101325 / 443948.52$ & $279.99 / 293.2$ & $1.41 / 5.98$ & $0 / 20.607$ & $-0.438 / 2.572$ \\
\hline Dynamic pressure, $\mathrm{Pa}$ & Shear stress, $\mathrm{Pa}$ & Specific heat, $\mathrm{J} /(\mathrm{kg} \cdot \mathrm{K})$ & Dynamic viscosity, Pa.s & Vorticity, $1 / \mathrm{s}$ \\
\hline $0 / 304.55$ & $0 / 4.85 \times 10^{-4}$ & $924.4 / 1921$ & $1.924 \times 10^{-5} / 2.005 \times 10^{-5}$ & $0.042 / 1599.554$ \\
\hline Prandtl number & Mach number & Fluid thermal conductivity, $\mathrm{W} /(\mathrm{m} \cdot \mathrm{K})$ & Turbulent viscosity, $\mathrm{Pa} \cdot \mathrm{s}$ & Turbulent time, $\mathrm{s}$ \\
\hline $0.6934402 / 1.4410829$ & $0 / 0.07$ & $0.0256 / 0.0263$ & $2.8616 \times 10^{-6} / 26.8015$ & $0.002 / 4.355$ \\
\hline Turbulent length, $\mathrm{m}$ & Turbulent energy, J/kg & Turbulent dissipation, $\mathrm{W} / \mathrm{kg}$ & Operative temperature, $\mathrm{K}$ & PMV \\
\hline $8 \times 10^{-4} / 0.174$ & $1.25 \times 10^{-6} / 7508.881$ & $2.87 \times 10^{-7} / 699174.33$ & $280.47 / 290.72$ & $-9 /-1.2$ \\
\hline \multicolumn{5}{|c|}{ Propane (liquid) } \\
\hline Pressure, $\mathrm{Pa}$ & Temperature, $\mathrm{K}$ & Density, $\mathrm{kg} / \mathrm{m}^{3}$ & Velocity, $\mathrm{m} / \mathrm{s}$ & Radial velocity, $\mathrm{m} / \mathrm{s}$ \\
\hline $101325 / 372265.02$ & $293.2 / 293.22$ & $503.35 / 503.37$ & $0 / 0.595$ & $-0.036 / 0.086$ \\
\hline Dynamic pressure, $\mathrm{Pa}$ & Shear stress, $\mathrm{Pa}$ & Specific heat, $\mathrm{J} /(\mathrm{kg} \cdot \mathrm{K})$ & Dynamic viscosity, $\mathrm{Pa} \cdot \mathrm{s}$ & Vorticity, $1 / \mathrm{s}$ \\
\hline $0 / 89.15$ & $0 / 4.18 \times 10^{-4}$ & $2685.3 / 2685.5$ & $0.0001 / 0.0001$ & $0.008 / 45.084$ \\
\hline Prandtl number & Mach number & Fluid thermal conductivity, $\mathrm{W} /(\mathrm{m} \cdot \mathrm{K})$ & Turbulent viscosity, $\mathrm{Pa} \cdot \mathrm{s}$ & Turbulent time, $\mathrm{s}$ \\
\hline $2.8568646 / 2.8569428$ & - & $0.096 / 0.096$ & $2.8616 \times 10^{-5} / 538.445$ & $0.018 / 37.527$ \\
\hline Turbulent length, m & Turbulent energy, J/kg & Turbulent dissipation, W/kg & Operative temperature, $\mathrm{K}$ & PMV \\
\hline $8 \times 10^{-4} / 0.266$ & $1.683 \times 10^{-8} / 53.925$ & $4.49 \times 10^{-10} / 244.68$ & $293.21 / 293.22$ & $-1.64 /-0.81$ \\
\hline
\end{tabular}




\begin{tabular}{|c|c|c|c|c|c|c|}
\hline Impact Factor: & $\begin{array}{l}\text { ISRA (India) } \\
\text { ISI (Dubai, UAE } \\
\text { GIF (Australia) } \\
\text { JIF }\end{array}$ & $\begin{array}{l}=1.344 \\
=0.829 \\
=0.564 \\
=1.500\end{array}$ & $\begin{array}{l}\text { SIS (USA) } \\
\text { PИНЦ (Russia } \\
\text { ESJI (KZ) } \\
\text { SJIF (Morocco }\end{array}$ & $\begin{array}{l}=0.912 \\
=0.207 \\
=4.102 \\
=\mathbf{2 . 0 3 1}\end{array}$ & $\begin{array}{l}\text { ICV (Poland) } \\
\text { PIF (India) } \\
\text { IBI (India) }\end{array}$ & $\begin{array}{l}=6.630 \\
=1.940 \\
=4.260\end{array}$ \\
\hline
\end{tabular}

\begin{tabular}{|c|c|c|c|c|}
\hline \multicolumn{5}{|c|}{ Propane (gas) } \\
\hline Pressure, $\mathrm{Pa}$ & Temperature, $\mathrm{K}$ & Density, $\mathrm{kg} / \mathrm{m}^{3}$ & Velocity, $\mathrm{m} / \mathrm{s}$ & Radial velocity, $\mathrm{m} / \mathrm{s}$ \\
\hline $101325 / 413190.84$ & $285.88 / 293.2$ & $1.91 / 7.58$ & $0 / 15.773$ & $-0.421 / 1.952$ \\
\hline Dynamic pressure, $\mathrm{Pa}$ & Shear stress, $\mathrm{Pa}$ & Specific heat, $\mathrm{J} /(\mathrm{kg} \cdot \mathrm{K})$ & Dynamic viscosity, Pa $\cdot \mathrm{s}$ & Vorticity, $1 / \mathrm{s}$ \\
\hline $0 / 238.88$ & $0 / 2.43 \times 10^{-4}$ & $1620.8 / 2397.6$ & $7.8284 \times 10^{-6} / 8.0081 \times 10^{-6}$ & $0.052 / 1221.953$ \\
\hline Prandtl number & Mach number & Fluid thermal conductivity, $\mathrm{W} /(\mathrm{m} \cdot \mathrm{K})$ & Turbulent viscosity, $\mathrm{Pa} \cdot \mathrm{s}$ & Turbulent time, $\mathrm{s}$ \\
\hline $0.7661103 / 1.1500248$ & $0 / 0.06$ & $0.0163 / 0.0168$ & $2.8616 \times 10^{-6} / 33.3446$ & $0.002 / 5.568$ \\
\hline Turbulent length, $\mathrm{m}$ & Turbulent energy, $\mathrm{J} / \mathrm{kg}$ & Turbulent dissipation, $\mathrm{W} / \mathrm{kg}$ & Operative temperature, $\mathrm{K}$ & PMV \\
\hline $8 \times 10^{-4} / 0.192$ & $7.646 \times 10^{-7} / 5281.459$ & $1.37 \times 10^{-7} / 359686.92$ & $286.15 / 291.48$ & $-6.18 /-0.98$ \\
\hline \multicolumn{5}{|c|}{ Propylene (gas) } \\
\hline Pressure, $\mathrm{Pa}$ & Temperature, $\mathrm{K}$ & Density, $\mathrm{kg} / \mathrm{m}^{3}$ & Velocity, $\mathrm{m} / \mathrm{s}$ & Radial velocity, $\mathrm{m} / \mathrm{s}$ \\
\hline $101325 / 415431.18$ & $284.72 / 293.2$ & $1.83 / 7.3$ & $0 / 16.369$ & $-0.425 / 2.023$ \\
\hline Dynamic pressure, $\mathrm{Pa}$ & Shear stress, $\mathrm{Pa}$ & Specific heat, $\mathrm{J} /(\mathrm{kg} \cdot \mathrm{K})$ & Dynamic viscosity, Pa's & Vorticity, $1 / \mathrm{s}$ \\
\hline $0 / 246.63$ & $0 / 2.58 \times 10^{-4}$ & $1503.1 / 2303.5$ & $8.1818 \times 10^{-6} / 8.4234 \times 10^{-6}$ & $0.036 / 1273.625$ \\
\hline Prandtl number & Mach number & Fluid thermal conductivity, $\mathrm{W} /(\mathrm{m} \cdot \mathrm{K})$ & Turbulent viscosity, $\mathrm{Pa} \cdot \mathrm{s}$ & Turbulent time, $\mathrm{s}$ \\
\hline $0.7867444 / 1.2382888$ & $0 / 0.06$ & $0.0152 / 0.0157$ & $2.8616 \times 10^{-6} / 31.7715$ & $0.002 / 5.345$ \\
\hline Turbulent length, $\mathrm{m}$ & Turbulent energy, J/kg & Turbulent dissipation, $\mathrm{W} / \mathrm{kg}$ & Operative temperature, $\mathrm{K}$ & PMV \\
\hline $8 \times 10^{-4} / 0.188$ & $8.298 \times 10^{-7} / 5517.442$ & $1.55 \times 10^{-7} / 395060.98$ & $285.03 / 291.24$ & $-6.67 /-1.04$ \\
\hline \multicolumn{5}{|c|}{ R22 (liquid) } \\
\hline Pressure, $\mathrm{Pa}$ & Temperature, $\mathrm{K}$ & Density, $\mathrm{kg} / \mathrm{m}^{3}$ & Velocity, $\mathrm{m} / \mathrm{s}$ & Radial velocity, $\mathrm{m} / \mathrm{s}$ \\
\hline $101325 / 216655.08$ & $293.2 / 293.2$ & $1212.34 / 1212.36$ & $0 / 0.247$ & $-0.015 / 0.035$ \\
\hline Dynamic pressure, $\mathrm{Pa}$ & Shear stress, $\mathrm{Pa}$ & Specific heat, $\mathrm{J} /(\mathrm{kg} \cdot \mathrm{K})$ & Dynamic viscosity, Pa $\cdot \mathrm{s}$ & Vorticity, $1 / \mathrm{s}$ \\
\hline $0 / 37.03$ & $0 / 2.26 \times 10^{-4}$ & $1236 / 1236.1$ & $0.0002 / 0.0002$ & $0.002 / 18.83$ \\
\hline Prandtl number & Mach number & Fluid thermal conductivity, $\mathrm{W} /(\mathrm{m} \cdot \mathrm{K})$ & Turbulent viscosity, $\mathrm{Pa} \cdot \mathrm{s}$ & Turbulent time, $\mathrm{s}$ \\
\hline $2.50384 / 2.5038692$ & - & $0.0857 / 0.0857$ & $2.8616 \times 10^{-5} / 537.5019$ & $0.044 / 90.382$ \\
\hline Turbulent length, $\mathrm{m}$ & Turbulent energy, $\mathrm{J} / \mathrm{kg}$ & Turbulent dissipation, W/kg & Operative temperature, $\mathrm{K}$ & PMV \\
\hline $8 \times 10^{-4} / 0.266$ & $2.902 \times 10^{-9} / 9.264$ & $3.21 \times 10^{-11} / 17.42$ & $293.2 / 293.2$ & $-1.2 /-0.82$ \\
\hline \multicolumn{5}{|c|}{ R123 (liquid) } \\
\hline Pressure, $\mathrm{Pa}$ & Temperature, $\mathrm{K}$ & Density, $\mathrm{kg} / \mathrm{m}^{3}$ & Velocity, $\mathrm{m} / \mathrm{s}$ & Radial velocity, $\mathrm{m} / \mathrm{s}$ \\
\hline $101325 / 196745.96$ & 293.2/293.21 & $1474.45 / 1474.48$ & $0 / 0.203$ & $-0.012 / 0.029$ \\
\hline Dynamic pressure, $\mathrm{Pa}$ & Shear stress, $\mathrm{Pa}$ & Specific heat, $\mathrm{J} /(\mathrm{kg} \cdot \mathrm{K})$ & Dynamic viscosity, Pa·s & Vorticity, $1 / \mathrm{s}$ \\
\hline $0 / 30.45$ & $0 / 2.96 \times 10^{-4}$ & 1013.6/1013.7 & $0.0004 / 0.0004$ & $0.002 / 15.423$ \\
\hline Prandtl number & Mach number & Fluid thermal conductivity, $\mathrm{W} /(\mathrm{m} \cdot \mathrm{K})$ & Turbulent viscosity, $\mathrm{Pa} \cdot \mathrm{s}$ & Turbulent time, $\mathrm{s}$ \\
\hline $5.7649858 / 5.7655009$ & - & $0.0778 / 0.0778$ & $2.8616 \times 10^{-5} / 536.8061$ & $0.054 / 109.925$ \\
\hline Turbulent length, $\mathrm{m}$ & Turbulent energy, $\mathrm{J} / \mathrm{kg}$ & Turbulent dissipation, $\mathrm{W} / \mathrm{kg}$ & Operative temperature, $\mathrm{K}$ & PMV \\
\hline $8 \times 10^{-4} / 0.266$ & $1.962 \times 10^{-9} / 6.247$ & $1.78 \times 10^{-11} / 9.65$ & $293.21 / 293.21$ & $-1.11 /-0.82$ \\
\hline \multicolumn{5}{|c|}{ R134a (liquid) } \\
\hline Pressure, $\mathrm{Pa}$ & Temperature, $\mathrm{K}$ & Density, $\mathrm{kg} / \mathrm{m}^{3}$ & Velocity, $\mathrm{m} / \mathrm{s}$ & Radial velocity, $\mathrm{m} / \mathrm{s}$ \\
\hline $101325 / 219273.67$ & $293.2 / 293.2$ & $1226.38 / 1226.4$ & $0 / 0.244$ & $-0.014 / 0.035$ \\
\hline Dynamic pressure, $\mathrm{Pa}$ & Shear stress, $\mathrm{Pa}$ & Specific heat, $\mathrm{J} /(\mathrm{kg} \cdot \mathrm{K})$ & Dynamic viscosity, Pa $\cdot s$ & Vorticity, $1 / \mathrm{s}$ \\
\hline $0 / 36.59$ & $0 / 2.43 \times 10^{-4}$ & $1405.2 / 1405.2$ & $0.0002 / 0.0002$ & $0.002 / 18.4$ \\
\hline Prandtl number & Mach number & Fluid thermal conductivity, $\mathrm{W} /(\mathrm{m} \cdot \mathrm{K})$ & Turbulent viscosity, $\mathrm{Pa} \cdot \mathrm{s}$ & Turbulent time, $\mathrm{s}$ \\
\hline $3.4989937 / 3.4990618$ & - & $0.0833 / 0.0833$ & $2.8616 \times 10^{-5} / 535.2238$ & $0.045 / 91.429$ \\
\hline Turbulent length, $\mathrm{m}$ & Turbulent energy, J/kg & Turbulent dissipation, $\mathrm{W} / \mathrm{kg}$ & Operative temperature, $\mathrm{K}$ & PMV \\
\hline $8 \times 10^{-4} / 0.266$ & $2.836 \times 10^{-9} / 8.976$ & $3.1 \times 10^{-11} / 16.61$ & $293.2 / 293.2$ & $-1.2 /-0.82$ \\
\hline \multicolumn{5}{|c|}{ RC318 (liquid) } \\
\hline Pressure, $\mathrm{Pa}$ & Temperature, $\mathrm{K}$ & Density, $\mathrm{kg} / \mathrm{m}^{3}$ & Velocity, $\mathrm{m} / \mathrm{s}$ & Radial velocity, $\mathrm{m} / \mathrm{s}$ \\
\hline $101325 / 199120.77$ & $293.2 / 293.2$ & $1517.39 / 1517.4$ & $0 / 0.197$ & $-0.011 / 0.028$ \\
\hline Dynamic pressure, $\mathrm{Pa}$ & Shear stress, $\mathrm{Pa}$ & Specific heat, $\mathrm{J} /(\mathrm{kg} \cdot \mathrm{K})$ & Dynamic viscosity, Pa $\cdot \mathrm{s}$ & Vorticity, $1 / \mathrm{s}$ \\
\hline $0 / 29.57$ & $0 / 2.69 \times 10^{-4}$ & $1101.3 / 1101.3$ & $0.0004 / 0.0004$ & $8.297 \times 10^{-4} / 14.836$ \\
\hline Prandtl number & Mach number & Fluid thermal conductivity, $\mathrm{W} /(\mathrm{m} \cdot \mathrm{K})$ & Turbulent viscosity, $\mathrm{Pa} \cdot \mathrm{s}$ & Turbulent time, $\mathrm{s}$ \\
\hline $6.4829169 / 6.4831606$ & - & $0.0664 / 0.0664$ & $2.8616 \times 10^{-5} / 533.7568$ & $0.056 / 113.124$ \\
\hline Turbulent length, $\mathrm{m}$ & Turbulent energy, $\mathrm{J} / \mathrm{kg}$ & Turbulent dissipation, W/kg & Operative temperature, $\mathrm{K}$ & PMV \\
\hline $8 \times 10^{-4} / 0.266$ & $1.852 \times 10^{-9} / 5.832$ & $1.64 \times 10^{-11} / 8.7$ & $293.2 / 293.2$ & $-1.1 /-0.82$ \\
\hline \multicolumn{5}{|c|}{ Water (liquid) } \\
\hline Pressure, $\mathrm{Pa}$ & Temperature, $\mathrm{K}$ & Density, $\mathrm{kg} / \mathrm{m}^{3}$ & Velocity, $\mathrm{m} / \mathrm{s}$ & Radial velocity, $\mathrm{m} / \mathrm{s}$ \\
\hline $101325 / 249149.99$ & $293.2 / 293.22$ & $997.56 / 997.56$ & $0 / 0.3$ & $-0.017 / 0.043$ \\
\hline Dynamic pressure, $\mathrm{Pa}$ & Shear stress, $\mathrm{Pa}$ & Specific heat, $\mathrm{J} /(\mathrm{kg} \cdot \mathrm{K})$ & Dynamic viscosity, $\mathrm{Pa} \cdot \mathrm{s}$ & Vorticity, $1 / \mathrm{s}$ \\
\hline $0 / 45$ & $0 / 6.53 \times 10^{-4}$ & $4184.4 / 4184.4$ & $0.001 / 0.001$ & $0.001 / 22.762$ \\
\hline Prandtl number & Mach number & Fluid thermal conductivity, $\mathrm{W} /(\mathrm{m} \cdot \mathrm{K})$ & Turbulent viscosity, $\mathrm{Pa} \cdot \mathrm{s}$ & Turbulent time, $\mathrm{s}$ \\
\hline $6.9916411 / 6.995035$ & - & $0.5985 / 0.5985$ & $2.8616 \times 10^{-5} / 534.1893$ & $0.037 / 74.37$ \\
\hline Turbulent length, $\mathrm{m}$ & Turbulent energy, $\mathrm{J} / \mathrm{kg}$ & Turbulent dissipation, W/kg & Operative temperature, $\mathrm{K}$ & PMV \\
\hline $8 \times 10^{-4} / 0.266$ & $4.286 \times 10^{-9} / 13.513$ & $5.76 \times 10^{-11} / 30.69$ & $293.21 / 293.22$ & $-1.29 /-0.81$ \\
\hline \multicolumn{5}{|c|}{ Xenon (gas) } \\
\hline Pressure, $\mathrm{Pa}$ & Temperature, $\mathrm{K}$ & Density, $\mathrm{kg} / \mathrm{m}^{3}$ & Velocity, $\mathrm{m} / \mathrm{s}$ & Radial velocity, $\mathrm{m} / \mathrm{s}$ \\
\hline $101325 / 244882.88$ & $281.04 / 293.24$ & $5.51 / 13.44$ & $0 / 5.8$ & $-0.348 / 0.644$ \\
\hline Dynamic pressure, $\mathrm{Pa}$ & Shear stress, $\mathrm{Pa}$ & Specific heat, $\mathrm{J} /(\mathrm{kg} \cdot \mathrm{K})$ & Dynamic viscosity, $\mathrm{Pa} \cdot \mathrm{s}$ & Vorticity, $1 / \mathrm{s}$ \\
\hline $0 / 95.92$ & $0 / 2.37 \times 10^{-4}$ & $162.9 / 417$ & $2.1641 \times 10^{-5} / 2.2512 \times 10^{-5}$ & $0.043 / 440.707$ \\
\hline Prandtl number & Mach number & Fluid thermal conductivity, $\mathrm{W} /(\mathrm{m} \cdot \mathrm{K})$ & Turbulent viscosity, $\mathrm{Pa} \cdot \mathrm{s}$ & Turbulent time, $\mathrm{s}$ \\
\hline $0.6558653 / 1.6834597$ & $0 / 0.04$ & $0.0053 / 0.0054$ & $2.8616 \times 10^{-6} / 44.9239$ & $0.004 / 9.705$ \\
\hline
\end{tabular}




\begin{tabular}{|c|c|c|c|c|}
\hline Turbulent length, $\mathrm{m}$ & Turbulent energy, J/kg & Turbulent dissipation, W/kg & Operative temperature, $\mathrm{K}$ & PMV \\
\hline $\mathbf{8} \times \mathbf{1 0}^{-4} / \mathbf{0 . 2 3 7}$ & $\mathbf{2 . 5 1 6} \times \mathbf{1 0} \mathbf{0}^{-7} / \mathbf{1 4 4 0 . 0 1 5}$ & $\mathbf{2 . 5 9 \times 1 0 ^ { - 8 } / 3 8 5 0 5 . 4 8}$ & $\mathbf{2 8 2 . 0 9 / 2 8 9 . 7 9}$ & $-\mathbf{7 . 1 4} / \mathbf{- 1 . 1 8}$ \\
\hline
\end{tabular}

Maximum specific heat is observed in gases having low density, such as hydrogen and helium. Specific heat and fluid thermal conductivity of water at room temperature are $4184.4 \mathrm{~J} /(\mathrm{kg} \cdot \mathrm{K})$ and 0.5985 $\mathrm{W} /(\mathrm{m} \cdot \mathrm{K})$, respectively. This is the highest values of these parameters for liquids and some gases.

The calculation of the Mach number was carried out only for gases. The Mach number for all gases is 0 at normal atmospheric pressure. The Mach number will be 0.2 when increasing of gas pressure in 10 times. Thermal processes in fluids can be represented by the Prandtl number. Value of the Prandtl number will depend on dynamic viscosity of liquid or gas. In accordance with the results written in the summary table, the highest thermal conductivity have liquids and, in particular, ethanol.

Transient flow of liquids and gases is represented by viscosity, energy, dissipation, and intensity of turbulent flow acted at the some distance for some time. Intensity of vorticity flow of gases prevails over intensity of vorticity flow of liquids. However, turbulent time of gas is less than liquid. Turbulent flow of liquid acts at the larger distance (length of the pipeline) than turbulent flow of gas. Consequently, energy and dissipation of turbulent flow of gas will be more than liquid.

\section{Conclusion}

Recommendations for this article will be fair for the straight pipelines of short length. At same mass flow rate it is necessary to increase in several times overall dimensions (diameters and wall thickness) of the pipeline for transportation of different gases. Dimensions of the pipeline it is possible to determine by the calculated values of fluid pressure. Flow of gases is accompanied by the most intensity of vorticity formation acted on the adopted section of the pipeline (on average $0.18 \mathrm{~m}$ ) not more than $10 \mathrm{~s}$.

\section{References:}

1. Chemezov D (2017) Calculation of pressure losses of liquid at a cylindrical straight pipeline section. ISJ Theoretical \& Applied Science, 12 (56): 19-22. Soi: http://s-o-i.org/1.1/TAS-1256-5 Doi: https://dx.doi.org/10.15863/TAS.2017.12.56.5

2. (2018) Gas-Liquid Flow. Available: http://www.thermopedia.com/content/2 (Accessed: 15.01.2018).

3. (2018) Chlorodifluoromethane. Available: https://en.wikipedia.org/wiki/Chlorodifluoromet hane (Accessed: 15.01.2018).

4. (2018) 2,2-Dichloro-1,1,1-trifluoroethane. Available: https://en.wikipedia.org/wiki/2,2-
Dichloro-1,1,1-trifluoroethane

(Accessed: 15.01.2018).

5. (2018) 1,1,1,2-Tetrafluoroethane. Available: https://en.wikipedia.org/wiki/1,1,1,2Tetrafluoroethane (Accessed: 15.01.2018).

6. (2018) RC-318 (Octafluorocyclobutane). Available:

http://www.wuzhouchem.com/cataloged/ods/rc318.htm (Accessed: 15.01.2018).

7. (2018) Thermal comfort. Available: https://en.wikipedia.org/wiki/Thermal_comfort (Accessed: 15.01.2018). 